\title{
Quasiparticle scattering at helium surfaces: A microscopic theory
}

\author{
M. B. Sobnack and J. C. Inkson \\ School of Physics, University of Exeter, Stocker Road, Exeter EX4 4QL, United Kingdom \\ J. C. H. Fung \\ Department of Mathematics, The Hong Kong University of Science and Technology, Clear Water Bay, Kowloon, Hong Kong, China
}

(Received 1 February 1999)

\begin{abstract}
We study the scattering of quasiparticles and atoms at the free surface of superfluid ${ }^{4} \mathrm{He}$ at $T=0 \mathrm{~K}$. As a starting point we take Beliaev's formalism in a real-space formulation and derive equations of motion for the elementary excitations valid in bulk helium, through the surface and the vacuum. We solve the equations numerically for the wave functions, from which we calculate the currents associated with the atoms, phonons, and rotons. These are then used to calculate the probabilities of all the possible one-to-one surface scattering processes. Results are presented for a wide range of energies, both for normal incidence and oblique incidence. The quantum evaporation results for fixed angles of incidence are used in experimental simulations which calculate the angular dependence of evaporated atom signals and these are compared with experimental data. [S0163-1829(99)05929-9]
\end{abstract}

\section{INTRODUCTION}

Ever since the experiments of Johnston and King, ${ }^{1}$ there has been a lot of interest in the subject of quantum evaporation from superfluid ${ }^{4} \mathrm{He}$. Johnston and $\mathrm{King}^{1}$ measured the velocity distribution of atoms evaporating from the free superfluid surface and reported that the vapor had a characteristic temperature of $1.6 \mathrm{~K}$ higher than the temperature $0.6 \mathrm{~K}$ of the superfluid. Anderson ${ }^{2}$ and Hyman $^{3}$ independently proposed that the result could be explained if the mechanism for evaporation, i.e., the conversion of quasiparticles at the free surface to atoms, were a one-to-one process. Anderson also pointed out that this quantum evaporation process should be the dominant one since the phase space available for processes involving more than one final state is very small in comparison.

Over the years, quantum evaporation and the reverse process of quantum condensation have been the subject of many studies, both experimental and theoretical. Several experiments $^{4-9}$ have investigated the interactions between the bulk quasiparticles (phonons, $R^{-}$rotons, and $R^{+}$rotons) and the free superfluid ${ }^{4} \mathrm{He}$ surface giving rise to evaporation. The time of flight experiments of Baird and Wyatt gave direct experimental evidence that the evaporation process is a quantum process. They produced phonons in bulk helium by generating minute heat pulses beneath the surface and detected signals due to evaporated atoms in the vapor. Analysis of the results showed that some of the phonons produced travelled ballistically to the surface where they ejected atoms on a one-to-one basis. Wyborn and Wyatt ${ }^{7}$ established that, together with the energy conservation

$$
\hbar \omega-\left|\mu_{0}\right|=\frac{\hbar^{2} \mathbf{k}^{2}}{2 m},
$$

where $\hbar \omega$ is the energy of the bulk quasiparticle and lies on the phonon-roton energy spectrum, $\mu_{0}(=-7.16 \mathrm{~K})$ is the chemical potential and $\hbar^{2} \mathbf{k}^{2} / 2 m\left[\mathbf{k}=\left(\mathbf{Q}, k_{a}\right)\right.$, where $k_{a}$ is the component of the atom wave vector normal to the surface] is the kinetic energy carried off by the evaporated atom, the momentum $\hbar \mathbf{Q}$ parallel to the surface is conserved because of the translational symmetry of the problem.

While quantum evaporation by phonons and $R^{+}$rotons has been confirmed by experiments ${ }^{5}$ over fourteen years ago, evaporation by the negative group velocity $R^{-}$rotons has only recently been observed experimentally. ${ }^{10}$ This has been attributed to the inability of the conventional heater to produce $R^{-}$rotons. Despite an ingenious method which got around the need for the heater to produce $R^{-}$rotons, Wyborn and $\mathrm{Wyatt}^{11}$ failed to observe quantum evaporation-they did observe a strong atom signal at the right place, but they no longer stand by the analysis of the results. ${ }^{10}$ Production of $R^{-}$rotons in the recent experiments ${ }^{10}$ has been achieved using a "cavity" heater.

Despite the considerable success of the experimental studies on quantum evaporation and quantum condensation, the probabilities of the different surface scattering processes cannot be determined experimentally using the present available techniques. One notable exception is the experiments of Edwards et al. ${ }^{12}$ who measured the reflectivity of atoms impinging on the free surface from the vapor. Their results showed that the probability of atoms reflecting from the surface is very low, but rises to 1 as the normal component of the atomic wave vector tends to 0 . The results also showed that most of the reflections are specular (i.e., elastic). Echenique and Pendry ${ }^{13,14}$ attempted to explain these reflectivity results in terms of the incident atoms coupling to a shower of quantized surface tension waves (ripplons) via the surface van der Waals potential, thereby losing most of their energy to the ripplons before penetrating the surface and hence cannot create any quasiparticles in bulk. However, in another series of experiments Edwards et al. ${ }^{15}$ showed that the oneto-one conversion of atoms to bulk states cannot be ruled out. Further Edwards and Fatouros ${ }^{16}$ showed that a much better fit to the reflectivity results than that of Echenique and Pendry can be obtained by neglecting ripplons altogether, 
and Swanson and Edwards ${ }^{17}$ predicted that the probability of not exciting any ripplons is quite high.

In contrast to the experimental successes, there has been relatively little quantitative theoretical work on the physical processes involved. The qualitative features of the physical processes involved can be understood on the basis of the surface acting as a strong, but elastic, scattering region which promotes mode conversion for quasiparticles and, on absorption, allows the helium atom to excite all the available quasiparticle channels. Together with the constraints imposed by the basic conservation laws, this approach can go a fair way towards qualitatively interpreting the experimental data. What is required for quantitative work is a microscopic theory of the dynamics of the helium surface scattering. It is clear that such a theory has to take into account (i) the nonlocality of the He-He interaction, (ii) the change in the properties of the condensate through the surface region, and (iii) the finite width of the surface. We include all these in the present study.

Although a great deal is known about the ground state and thermodynamic properties of both the bulk and the surface liquid helium, the problem of the scattering of bulk excitations at or through the surface has not received a lot of attention. As mentioned above, Echenique and Pendry ${ }^{13,14}$ and later Edwards et al. ${ }^{15}$ studied the problem of the surface scattering in helium on the basis of ripplon interactions. Caroli et al. ${ }^{18,19}$ attempted a microscopic approach, but were only able to discuss the qualitative features of the transmission associated with a multichannel system. Sasaki and Kunimasa ${ }^{20}$ looked at the interface scattering problem with a semiempirical coupling model. In all the above, however, the structure of the surface was largely ignored. Other studies $^{3,21,22}$ used a perturbation method in which, contrary to experimental evidence, ${ }^{12}$ the quasiparticles are weakly coupled to the atoms. Further, since the liquid and the vapor are comprised of the same material, the distinction between liquid and vapor operators made in these tunnelling Hamiltonian approaches is not appropriate. Maris ${ }^{23}$ used an "adiabatic" model and found that quasiparticles (phonons and rotons) with energies between the roton minimum energy $\Delta$ $\sim 8.7 \mathrm{~K}$ and the maxon energy $\Delta_{m} \sim 13.9 \mathrm{~K}$ do not evaporate atoms, whereas experiments ${ }^{6,24}$ show that $R^{+}$rotons with these energies do contribute to quantum evaporation.

The microscopic theory of Mulheran and Inkson predicted scattering rates for all the one-to-one surface processes. ${ }^{25}$ They wrote down an equation for the inhomogeneous ${ }^{4} \mathrm{He}$ system which they solved in a local density approximation. They found solutions to the local-density-equation using the Wentzel-Kramers-Brillouin (WKB) formalism. They took the difference between the exact interaction term and the local-density-interaction as a perturbation potential and, using a first-order perturbation theory expansion, calculated the matrix elements, and hence the probabilities, for the various transitions between the quantum states associated with the WKB solutions. Their results were in broad agreement with experimental observations, but were, however, restricted to normal incidence $\left(\mathbf{Q}=\mathbf{0} \AA^{-1}\right)$.

Dalfovo and co-workers ${ }^{26-30}$ have recently produced scattering rates for the surface processes using a linearized density functional theory with their phenomenological "OrsayTrento" density functional. They gave results for both normal incidence and oblique incidence. In particular they found that $R^{-}$rotons evaporate atoms only at energies and parallel momenta which exclude phonons from the surface scattering processes, a result which is in contrast with the recent experiments of Tucker and Wyatt. ${ }^{31}$ They also found that the probabilities do not depend on the angle of incidence of the incoming excitation.

The present study removes the WKB and normalincidence restrictions in the Mulheran and Inkson ${ }^{25}$ theory. We neglect inelastic processes and assume that all the quasiparticles are stable and travel ballistically. We investigate the one-to-one scattering processes at bulk energies $\hbar \omega$ covering the range from just above the binding energy $\left|\mu_{0}\right|$ $=7.16 \mathrm{~K}$ to energies higher than the maxon energy $\Delta_{m}$ $\sim 13.9$ K. Preliminary results have been reported earlier. ${ }^{32,33}$

The paper is organized as follows. In Sec. II, we present the method we use and derive the equations of motion; the solutions to the equations are presented in Sec. III. We also give our calculated scattering rates, both for normal incidence and oblique incidence (for both fixed parallel momenta and fixed angles of incidence) and compare them with those published and those from experimental simulations. We conclude in Sec. IV with some remarks and discuss some possible future work.

\section{FORMALISM}

The low-temperature properties of bulk superfluid ${ }^{4} \mathrm{He}$ are well understood, at a phenomenological level, in terms of Landau's picture of a weakly interacting quasiparticle gas of phonons and rotons. At a more microscopic level, one has to take into account the coupling between the condensate and the elementary excitations and use field-theoretic analysis, built on the fundamental role of Bose-broken symmetry - the superfluid phase coincides with the appearance of a macroscopic wave function. This approach was initiated by the work of Bogoliubov. ${ }^{34}$ Beliaev $^{35}$ and, a year later, Hugenholtz and Pines ${ }^{36}$ formulated it in a systematic way. In this study we adapt Beliaev's microscopic theory to the inhomogeneous problem of quasiparticle scattering at the free surface of superfluid ${ }^{4} \mathrm{He}$.

Beliaev's theory for the bulk superfliud uses many-body Green's function techniques to study the properties of the quasiparticles of the system. It is based on Bogoliubov's assumptions that the condensate (the state of zero momentum) is macroscopically occupied and that the excited states are dominated by scatterings involving two condensate particles. With these assumptions, Beliaev found that the Feynman diagram equations [see Fig. 1(a)] for the system contain three irreducible self-energy diagrams (which stem from the interaction of particles not in the condensate with those in the condensate). $\Sigma_{11}$ is the sum of all self-energy diagrams with equal numbers of incoming and outgoing condensate lines (dotted lines); $\Sigma_{02}$ is the sum of all self-energy diagrams in which the number of incoming condensate lines exceeds the number of outgoing ones by 2 , while $\Sigma_{20}$ has two more outgoing condensate lines than incoming ones. These selfenergies have to be inserted in the equations in such a way that the total number of particles (condensate + noncondensate) are conserved. The superfluid ${ }^{4} \mathrm{He}$ system has two propagators $G(\mathbf{k}, \omega)$ and $F(\mathbf{k}, \omega)$ : the usual single- 


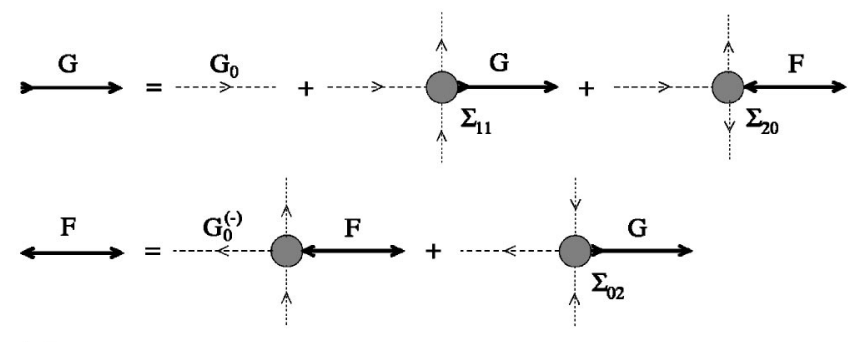

(a)

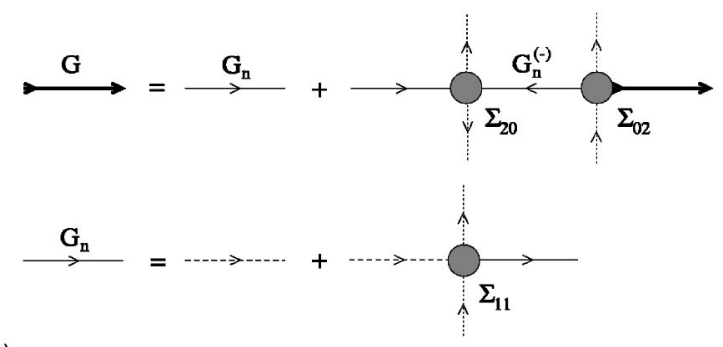

(b)

FIG. 1. (a) Feynman diagrams for the superfluid helium system [after Beliaev (Ref. 35)]. (b) Alternative way of constructing the diagram for $G$ using an intermediate "normal state" Green's function $G_{n}$ (see, for example, Pines ${ }^{37}$ ).

particle Green's function $G(\mathbf{k}, \omega)$ and the "anomalous", Green's function $F(\mathbf{k}, \omega)$, which describes the effects associated with a quasiparticle propagating in a correlated system. $G_{0}(\mathbf{k}, \omega)$ is the free-particle Green's function.

The top equation for $G$ can be split into two equations [see Fig. 1(b)] by the introduction of a "normal state", intermediate Green's function $G_{n}(\mathbf{k}, \omega)$ (see, for example, Pines ${ }^{37}$ ). In the low-density (Bogoliubov) limit, the firstorder diagrams for the irreducible self-energies give

$$
\Sigma_{11}=\rho V(0)+\rho V(\mathbf{k}) \quad \text { and } \quad \Sigma_{02}=\Sigma_{20}=\rho V(\mathbf{k}) \text {, }
$$

where $V(\mathbf{k})$ is the Fourier transform of the helium-helium potential and $\rho$ the condensate density. $G$ and $F$ then have poles at $\hbar \omega= \pm E_{B}$, where $E_{B}$ is the Bogoliubov spectrum ${ }^{34}$

$$
E_{B}(\mathbf{k})=\left[\frac{\hbar^{4} \mathbf{k}^{4}}{4 m^{2}}+2 \rho \frac{\hbar^{2} \mathbf{k}^{2}}{2 m} V(\mathbf{k})\right]^{1 / 2}
$$

From Fig. 1(b) in real space one can write down the equation of motion in this limit as

$$
\left[\hbar \omega-\mu(\mathbf{r})+\frac{\hbar^{2}}{2 m} \nabla^{2}\right] \phi(\mathbf{r})-\int_{-\infty}^{+\infty} \Sigma\left(\mathbf{r}, \mathbf{r}^{\prime}, \omega\right) \phi\left(\mathbf{r}^{\prime}\right) d^{3} \mathbf{r}^{\prime}=0
$$

for the "particle-hole" wave function $\phi(\mathbf{r})$ valid in bulk, through the surface and in the vacuum. The function $\mu(\mathbf{r})$, defined at the end of this section, changes from 0 (in bulk) to $\left|\mu_{0}\right|$ (in the vacuum) across the surface and $\mu_{0}$ is the condensate chemical potential. The central feature of this equation is the self-energy

$$
\begin{aligned}
\Sigma\left(\mathbf{r}, \mathbf{r}^{\prime}, \omega\right)= & \sqrt{\rho(\mathbf{r}) \rho\left(\mathbf{r}^{\prime}\right)} V\left(\mathbf{r}-\mathbf{r}^{\prime}\right) \\
& +\sqrt{\rho(\mathbf{r}) \rho\left(\mathbf{r}^{\prime}\right)} \iint_{-\infty}^{+\infty} \sqrt{\rho\left(\mathbf{r}_{1}\right)} V\left(\mathbf{r}-\mathbf{r}_{1}\right) \\
& \times G_{n}\left(\mathbf{r}_{1}-\mathbf{r}_{2},-\omega\right) V\left(\mathbf{r}_{2}-\mathbf{r}^{\prime}\right) \sqrt{\rho\left(\mathbf{r}_{2}\right)} d^{3} \mathbf{r}_{1} d^{3} \mathbf{r}_{2},
\end{aligned}
$$

where $G_{n}(\mathbf{r}, \omega)$ is the "normal" Green's function. ${ }^{37}$ The self-energy is a function of the condensate density $\rho(\mathbf{r})$ and the nonlocal helium-helium interaction $V\left(\mathbf{r}-\mathbf{r}^{\prime}\right)$. The "hole-particle", wave function $\psi(\mathbf{r})$, associated with the Green's function $F$, is obtained from the corresponding equation for $F$ in Fig. 1(a) as

$$
\begin{aligned}
\psi(\mathbf{r})= & \sqrt{\rho(\mathbf{r})} \iint_{-\infty}^{+\infty} G_{n}\left(\mathbf{r}-\mathbf{r}_{1},-\omega\right) \\
& \times V\left(\mathbf{r}_{1}-\mathbf{r}^{\prime}\right) \sqrt{\rho\left(\mathbf{r}^{\prime}\right)} \phi\left(\mathbf{r}^{\prime}\right) d^{3} \mathbf{r}_{1} d^{3} \mathbf{r}^{\prime} .
\end{aligned}
$$

The "hole-particle" wave function $\psi(\mathbf{r})$ is necessary to correctly describe the effects associated with a quasiparticle propagating through a correlated system. In the bulk, along the lower part of phonon branch of the excitation spectrum $\psi(\mathbf{r})=O[\phi(\mathbf{r})] ; \psi(\mathbf{r})=o[\phi(\mathbf{r})]$ along the roton branch (near the roton threshold $\Delta \sim 8.7 \mathrm{~K}$ ) and $\psi(\mathbf{r}) \rightarrow 0$ at very high $\hbar \omega \gg \Delta$. In the vacuum, $\psi(\mathbf{r})$ vanishes identically.

In deriving the above equations, we have allowed the condensate density $\rho(\mathbf{r})$ to vary with position so that Eqs. (3) and (4) may be used to tackle the general inhomogeneous problem such as the free surface. We do not attempt to solve for the density variation, but rather use this as an input to the calculation. To solve for the density variation would require a solution to the ground state problem. This is both complicated and difficult and not relevant to the immediate problem we are considering. Deep in bulk the density has the value of bulk superfluid condensate, i.e., $\rho=$ const, and high above the surface it has the vacuum value $\rho=0$. Equation (3) has the expected limits - in bulk it is the Schrödinger equation for the quasiparticles (of energy $\hbar \omega$ ) and in the vacuum it is the Schrödinger equation for the free atom (of energy $\hbar \omega$ $\left.-\left|\mu_{0}\right|\right)$.

From the diagrams in Fig. 1(a), one can also write the pair of coupled equations

$$
\begin{aligned}
& {\left[\hbar \omega-\mu(\mathbf{r})+\frac{\hbar^{2}}{2 m} \nabla^{2}\right] \phi(\mathbf{r})-\sqrt{\rho(\mathbf{r})} \int_{-\infty}^{+\infty} \sqrt{\rho\left(\mathbf{r}^{\prime}\right)} V\left(\mathbf{r}-\mathbf{r}^{\prime}\right)} \\
& \times\left[\phi\left(\mathbf{r}^{\prime}\right)+\psi\left(\mathbf{r}^{\prime}\right)\right] d^{3} \mathbf{r}^{\prime}=0, \\
& {\left[-\hbar \omega+\mu(\mathbf{r})+\frac{\hbar^{2}}{2 m} \nabla^{2}\right] \psi(\mathbf{r})-\sqrt{\rho(\mathbf{r})} \int_{-\infty}^{+\infty} \sqrt{\rho\left(\mathbf{r}^{\prime}\right)} V\left(\mathbf{r}-\mathbf{r}^{\prime}\right)} \\
& \times\left[\phi\left(\mathbf{r}^{\prime}\right)+\psi\left(\mathbf{r}^{\prime}\right)\right] d^{3} \mathbf{r}^{\prime}=0
\end{aligned}
$$

for $\phi(\mathbf{r})$ and $\psi(\mathbf{r})$ as an alternative to Eqs. (3) and (4). We note that the above equations have the appearance of onebody Schrödinger equations with a nonlocal potential, reflecting that this is a many-body problem. Again in deriving these equations, we have allowed the condensate density to vary with position.

Because of the symmetry of the problem, the density profile $\rho(\mathbf{r})$ depends only on $z$, the component of $\mathbf{r}=(\mathbf{R}, z)$ normal to the interface, i.e., $\rho=\rho(z)$. We give the quasiparticles 
a momentum $\hbar \mathbf{Q}$ parallel to the surface. Since the elementary excitations conserve the planar symmetry, the parallel momentum is conserved and we can look for solutions of the form

$$
\phi(\mathbf{r})=e^{i \mathbf{Q} \cdot \mathbf{R}} \phi(z) \quad \text { and } \quad \psi(\mathbf{r})=e^{i \mathbf{Q} \cdot \mathbf{R}} \psi(z),
$$

where $\mathbf{R}=(x, y)$. This reduces Eqs. (3) and (4) to the onedimensional equations

$$
\begin{array}{r}
{\left[\hbar \omega-\mu(z)-\frac{\hbar^{2} \mathbf{Q}^{2}}{2 m}+\frac{\hbar^{2}}{2 m} \frac{d^{2}}{d z^{2}}\right] \phi(z)} \\
-\int_{-\infty}^{+\infty} \Sigma\left(z, z^{\prime}, \omega, \mathbf{Q}\right) \phi\left(z^{\prime}\right) d z^{\prime}=0
\end{array}
$$

and

$$
\begin{aligned}
\psi(z)= & \sqrt{\rho(z)} \iint_{-\infty}^{+\infty} G_{n}\left(z-z_{1},-\omega, \mathbf{Q}\right) \\
& \times V\left(\mathbf{Q}, z_{1}-z^{\prime}\right) \sqrt{\rho\left(z^{\prime}\right)} \phi\left(z^{\prime}\right) d z_{1} d z^{\prime},
\end{aligned}
$$

respectively, and the coupled equations (5) to

$$
\begin{aligned}
& {\left[\hbar \omega-\mu(z)-\alpha \mathbf{Q}^{2}+\alpha \frac{d^{2}}{d z^{2}}\right] \phi(z)-\sqrt{\rho(z)}} \\
& \times \int \sqrt{\rho\left(z^{\prime}\right)} V\left(\mathbf{Q}, z-z^{\prime}\right)\left[\phi\left(z^{\prime}\right)+\psi\left(z^{\prime}\right)\right] d z^{\prime}=0, \\
& {\left[-\hbar \omega+\mu(z)-\alpha \mathbf{Q}^{2}+\alpha \frac{d^{2}}{d z^{2}}\right] \psi(z)-\sqrt{\rho(z)}} \\
& \times \int \sqrt{\rho\left(z^{\prime}\right)} V\left(\mathbf{Q}, z-z^{\prime}\right)\left[\phi\left(z^{\prime}\right)+\psi\left(z^{\prime}\right)\right] d z^{\prime}=0,
\end{aligned}
$$

where $\alpha=\hbar^{2} / 2 m$. We assume that the interaction is the same as in bulk helium and use the effective potential of Brueckner and Sawada. ${ }^{38}$ The Brueckner potential gives a good fit to the experimentally observed quasiparticle excitation spectrum in bulk when used with the Bogoliubov spectrum (2) and keeps the non-locality of the $\mathrm{He}-\mathrm{He}$ interaction. The Brueckner potential takes the form

$$
\begin{aligned}
V\left(\mathbf{Q}, z-z^{\prime}\right) & =\frac{V_{0}}{\pi} \int_{0}^{+\infty} \frac{\sin a_{0} \sqrt{\mathbf{Q}^{2}+q^{2}}}{\sqrt{\mathbf{Q}^{2}+q^{2}}} \cos q\left(z-z^{\prime}\right) d q \\
& = \begin{cases}\left(V_{0} / 2\right) J_{0}\left(|\mathbf{Q}| \sqrt{a_{0}^{2}-\left(z-z^{\prime}\right)^{2}}\right) & \text { for }\left|z-z^{\prime}\right| \leqslant a_{0}, \\
0 & \text { otherwise, }\end{cases}
\end{aligned}
$$

where $J_{0}$ is the Bessel function of the first kind of order zero, reflecting the cylindrical symmetry associated with the translational symmetry of the problem. $V_{0}=17.0 \mathrm{~K}^{-1}$ and $a_{0}$ $=2.26 \AA^{38}{ }^{38}$ For normal incidence, $\mathbf{Q}=\mathbf{0} \AA^{-1}$, the potential reduces to the top hat potential $V\left(z-z^{\prime}\right)=V_{0} / 2$ for $\left|z-z^{\prime}\right|$ $\leqslant a_{0}$ and 0 elsewhere.

The variation of the chemical potential is described as $\mu(z)=\left|\mu_{0}\right| f(z)$. The choice of $f(z)$ is required to model the change in $\mu$ across the surface. We have used both the forms $f(z)=[1-\rho(z)] \quad$ and $\quad f(z)=\left(2 \nu a_{0}\right)^{-1} \ln \{[1+\exp \nu(z$ $\left.\left.\left.+a_{0}\right)\right] /\left[1+\exp \nu\left(z-a_{0}\right)\right]\right\}$ (with $\nu$ chosen to give a sharper change than the first form); both forms give comparable results. We use a Fermi function for the surface profile $\rho(z)$.

\section{SOLUTION OF THE EQUATIONS AND RESULTS}

To calculate the wave functions $\phi(z)$ and $\psi(z)$, we either solve the full equation (6) numerically for $\phi(z)$ and then evaluate $\psi(z)$ from Eq. (7), or numerically solve the pair of coupled equations (8). In either case, we do this in a box of size $2 L(z=-L-s$ to $z=L-s$, with the surface centered at $z=0$ and with bulk helium in $z<0$ ); we look for (real) standing wave solutions. We take the surface to have a 90 $10 \%$ width of $6.5 \AA$ which is within the accepted experimental estimate. ${ }^{39}$ Depending on $\hbar \omega$ and $\hbar \mathbf{Q}$, one or more quasiparticles may be excluded from the surface scattering processes by conservation of energy and momentum parallel to the surface, but there are normally insufficient constraints to solve for the general solution given particular system parameters. To calculate the various scattering probabilities therefore, we always need several solutions at the same energy and parallel momentum. These are obtained by keeping $\hbar \omega$ and $\hbar \mathbf{Q}$ fixed and solving the equation for different values of $s$.

To illustrate the process of obtaining the scattering parameters from the numerical solutions, Figs. 2(a) and 2(b) show the calculated wave functions (solid lines) at $\hbar \omega=12.3 \mathrm{~K}$, $|\mathbf{Q}|=0.75 \AA^{-1}$. At this parallel momentum, simple conservation of energy and momentum shows that atoms cannot be emitted for $\hbar \omega<10.6 \mathrm{~K}$ and phonons are excluded from the surface processes for $\hbar \omega<12.8 \mathrm{~K}$. At the energy and parallel momentum shown, in bulk, $\phi(z)$ has two Fourier components $k_{-}, k_{+}$, corresponding to $R^{-}$rotons and $R^{+}$rotons, respectively, at precisely the normal wave vectors expected from the excitation spectrum. In the vacuum it has a single component $k=k_{a}$ in agreement with the value for emitted $\mathrm{He}$ atoms calculated from $\hbar \omega=\left|\mu_{0}\right|+\hbar^{2}\left(k_{a}^{2}+\mathbf{Q}^{2}\right) / 2 m . \phi(z)$ can be fitted with functions of the form

$$
\begin{gathered}
\phi(z<0)=\sum_{i=-,+} \phi_{i} \cos \left(k_{i} z+\theta_{i}\right), \\
\phi(z>0)=\phi_{a} \cos \left(k_{a} z+\theta_{a}\right),
\end{gathered}
$$

representing the bulk $(z<0)$ and the vacuum $(z>0)$ limiting wave functions, respectively. $\theta_{i}(i=a,-,+)$ is a phase. The 

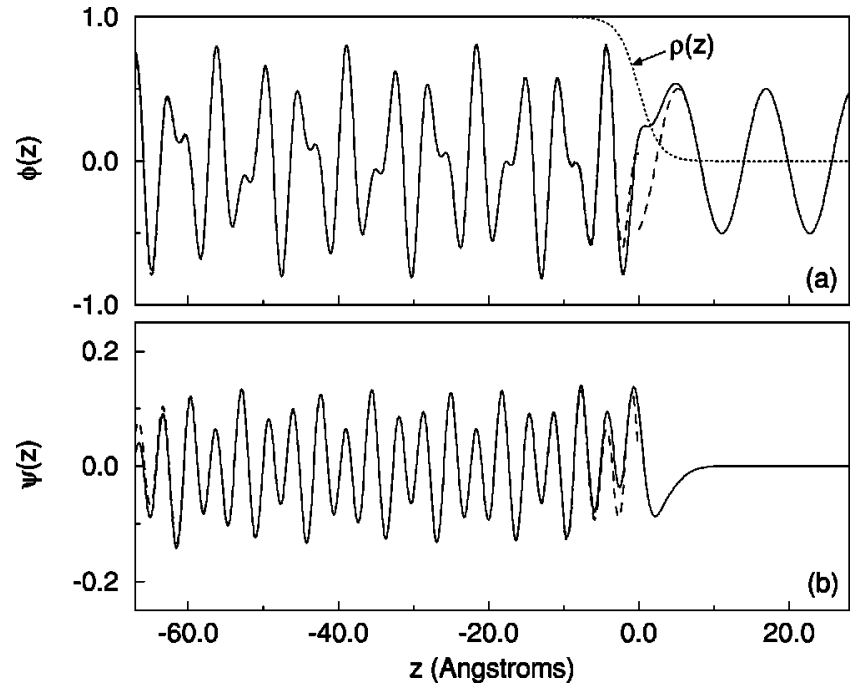

FIG. 2. The wave functions $\phi(z)$ (a) and $\psi(z)$ (b) at $\hbar \omega$ $=12.3 \mathrm{~K} .|\mathbf{Q}|=0.75 \AA^{-1}$. The short-dashed line is the surface profile. The surface is centered at $z=0(L=48 \AA$ and $s=20 \AA)$. The long-dashed lines are the numerical fit to the wave functions.

"hole-particle" wave function $\psi(z)$ can be similarly fitted remembering that, in the vacuum, $\psi_{a}=0$. The fits are shown as long-dashed lines in the figures.

The wave vectors $k_{i}$, phases $\theta_{i}$, (real) amplitudes $\phi_{i}$ and $\psi_{i}(i=a,-,+)$ are extracted from the fits. The amplitudes $\phi_{i}$ and $\psi_{i}$ are then used to calculate the current $j_{i}$ associated with each quasiparticle or atom from

$$
j_{i}=\frac{1}{2} \mathbf{v}_{i}^{g}\left(\phi_{i}^{2}-\psi_{i}^{2}\right)
$$

where $\mathrm{v}_{i}^{g}(i=a,-,+)$ is the group velocity of the elementary excitation $i$. From these currents we calculate the various scattering probabilities. We do not give details here as they have been fully discussed elsewhere. ${ }^{26,27,32}$

The scattering probabilities from state $i$ to state $j, P_{i j}$, have to satisfy the time reversal symmetry condition $P_{i j}$ $=P_{j i}$ and, the "scattering matrix" being unitary, the unitarity condition that

$$
\sum_{i} P_{i j}=1
$$

for each $j$. These two conditions provide a useful check on the calculated probabilities. We give below a summary of our calculated probability results. The calculated probabilities satisfy, to within numerical accuracy, both the timereversal symmetry condition and the unitarity condition.

\section{A. Normal incidence}

In this section, the elementary excitations are incident normal to the surface, $\mathbf{Q}=\mathbf{0} \AA^{-1}$. For energies $\hbar \omega<\Delta$ $\sim 8.7 \mathrm{~K}$, the roton minimum energy, only phonons are present in bulk and the only quasiparticles present at energies $\hbar \omega>\Delta_{m} \sim 13.9 \mathrm{~K}$, the maxon energy, are $R^{+}$rotons. At energies between the roton minimum and the maxon all three bulk quasiparticles (phonons, $R^{-}$rotons, and $R^{+}$rotons) take part in the surface scattering processes.

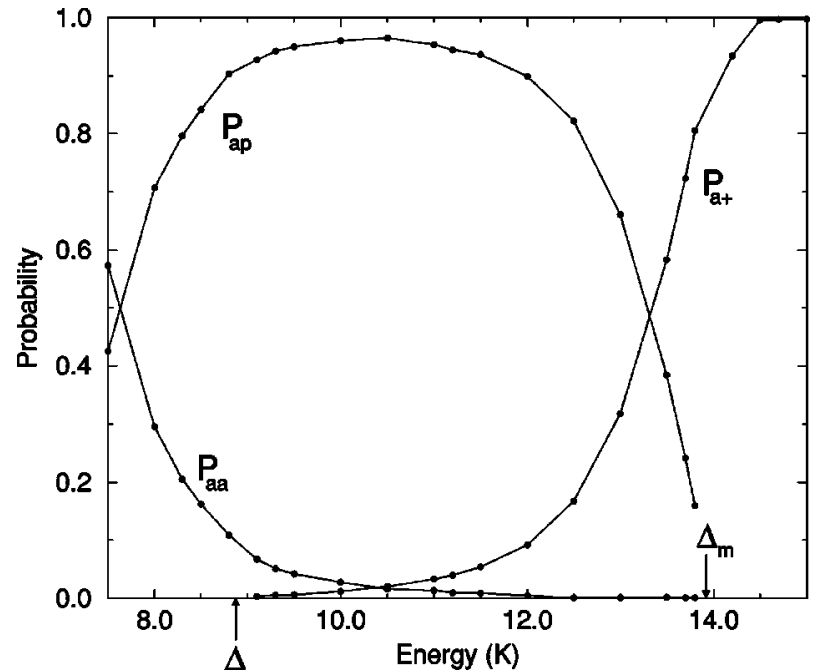

FIG. 3. The various scattering probabilities $P_{a j}$ as a function of bulk energy for an atom incident on the surface. $\Delta$ and $\Delta_{m}$ are, respectively, the roton minimum energy and the maxon energy. $P_{a a}:$ reflection of a ${ }^{4} \mathrm{He}$ atom from the surface, $P_{a p}:$ absorption of a ${ }^{4} \mathrm{He}$ atom into a phonon mode, and $P_{a+}$ : absorption of a ${ }^{4} \mathrm{He}$ atom into an $R^{+}$roton mode. $P_{a-}=0$.

Figures 3, 4, and 5 show, respectively, the calculated probabilities as a function of bulk energy for the possible transitions available to atoms, phonons, and $R^{+}$rotons incident to the surface at normal incidence. The results can most easily be understood in terms of the adiabatic model of Mulheran and Inkson. ${ }^{25}$ In their WKB-based approaches, through the surface atom states transform adiabatically into phonon states and vice versa, whereas $R^{-}$roton and $R^{+}$roton states form standing waves limited to the condensate. Transitions between these WKB states take place in the surface region. This means that an incoming atom primarily condenses as a phonon below the roton minimum but as an $R^{+}$roton above the maxon, and $R^{+}$rotons above the maxon and phonons below the roton minimum evaporate. Between the roton minimum and the maxon, an $R^{+}$roton scatters as an $R^{-}$

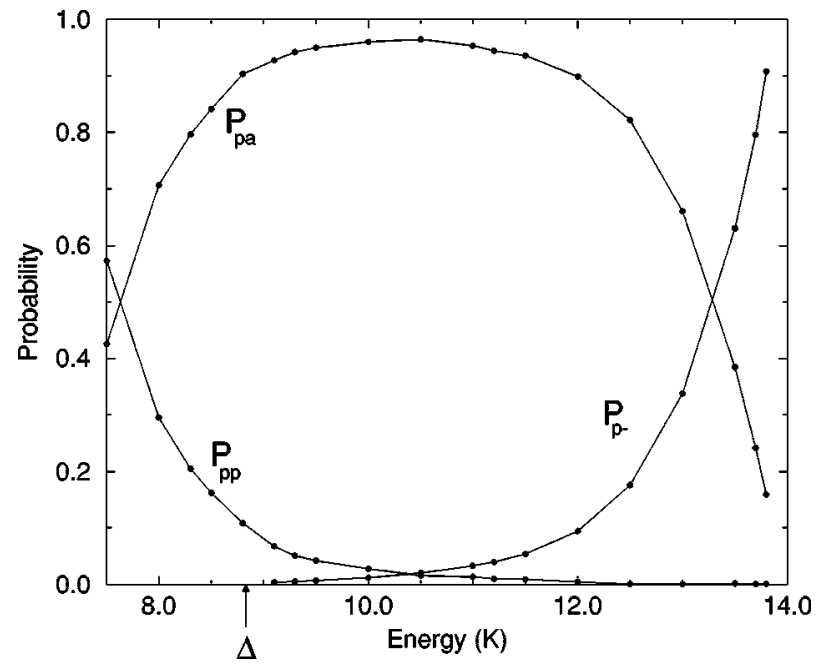

FIG. 4. The probabilities $P_{p j}$ as a function of energy for an incident phonon. $P_{p p}$ : reflection of a phonon from the surface, $P_{p a}$ : evaporation of a ${ }^{4} \mathrm{He}$ atom by a phonon, and $P_{p-}:$ reflection of a phonon mode into an $R^{-}$roton mode. $P_{p+}=0$. 


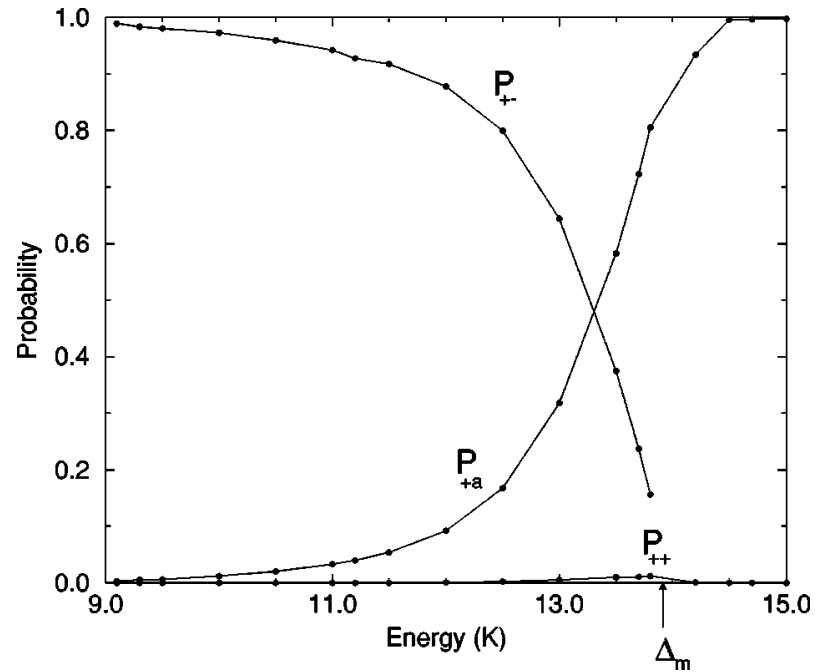

FIG. 5. The transition probabilities for an incident $R^{+}$roton as a function of energy. $P_{++}$: reflection of an $R^{+}$roton from the surface, $P_{+a}$ : evaporation of a ${ }^{4} \mathrm{He}$ atom by an $R^{+}$roton, and $P_{+-}$: reflection of an $R^{+}$roton into an $R^{-}$roton. $P_{+p}=0$.

roton and vice versa and the coupling between the WKB states varies according to the overlap between the states in the surface and hence primarily the closeness of the momentum values.

The results shown in Figs. 3, 4, and 5 follow very much this pattern. The atomic reflectivity $P_{a a}$ is about 0.58 at a bulk energy just above the binding energy $\left|\mu_{0}\right|$, which corresponds to atoms with (normal) wave vector $k_{a} \rightarrow 0$, and decreases rapidly to 0 with energy (Fig. 3). This is in agreement with the experimental results of Edwards et al. ${ }^{12}$ The measured $P_{a a}$, however, decreases faster with energyprobably due to inelastic phonon excitation processes. The calculated phonon reflectivity $P_{p p}$ is equal to $P_{a a}$ as a consequence of unitarity and time-reversal symmetry for energies between $\left|\mu_{0}\right|$ and the roton minimum $\Delta$. It would also be affected by the inclusion of inelastic processes. The calculated (and experimental) atomic reflectivities are continuous across the roton minimum $\Delta$. These results differ both quantitatively and qualitatively from those of Stingari et al. ${ }^{28}$ and Guilleumas et al. ${ }^{30}$ who found that, at normal incidence, $P_{a a}=P_{p p}=0$, even at bulk energies just above $\left|\mu_{0}\right|$, contrary to simple quantum mechanical considerations.

Atoms condense to phonons with probability $P_{a p} \sim 0.42$ at a bulk energy just above the binding energy (Fig. 3). $P_{a p}$ increases with energy to a maximum of about 0.9 around $\hbar \omega \sim 10.5 \mathrm{~K}$ and then decreases monotonically to zero as the energy reaches the maxon energy, the transition to $R^{+}$rotons taking over as expected from the Mulheran and Inkson model. ${ }^{25}$ The probability $P_{p a}\left(=P_{a p}\right)$ of the converse process of quantum evaporation by phonons has the same energy dependence (Fig. 4). Again these results are in contrast with those of Stingari et al. ${ }^{28}$ and Guilleumas et al. ${ }^{30}$ who found that $P_{a p}=P_{p a} \rightarrow 1$ as $\hbar \omega \rightarrow\left|\mu_{0}\right|$ and decrease monotonically to zero towards the maxon. The dominant scattering process for incoming phonons at energies near the maxon is the mode change reflection to $R^{-}$rotons, and for incoming $R^{-}$rotons the reverse reflection $R^{-}$rotons to phonons, with $P_{p-}=P_{-p}$.

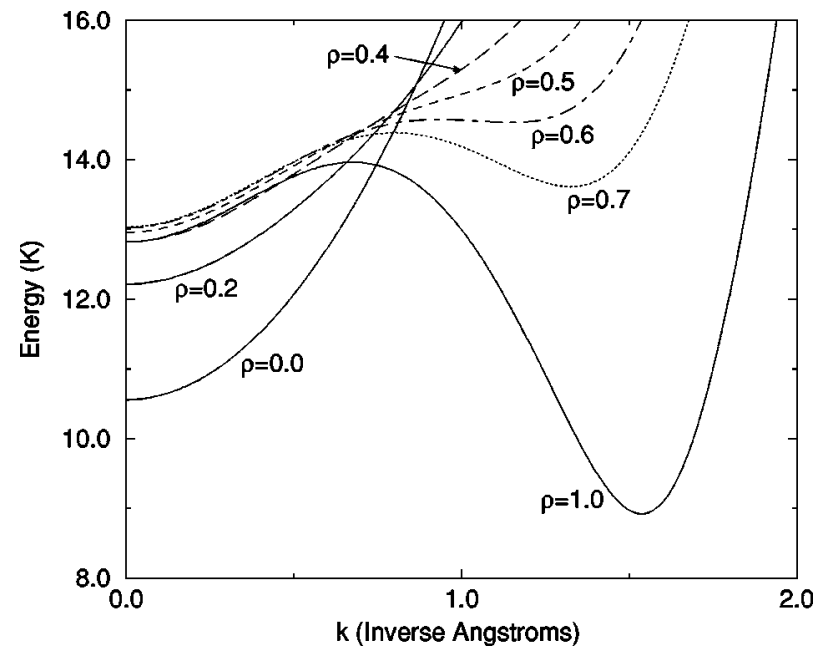

FIG. 6. The quasiparticle spectrum as a function of the normal component $k$ of the wave vector for various densities $\rho$ between $\rho$ $=1$ (bulk) and $\rho=0$ (vacuum). $|\mathbf{Q}|=0.75 \AA^{-1}$.

For incoming $R^{+}$rotons (Fig. 5), the dominant process at energies just above the roton threshold $\Delta$ is reflection as $R^{-}$ rotons. As the energy of the incoming $R^{+}$roton increases to the maxon, the probability $P_{+-}$of the process decreases to zero. There the main scattering process is the quantum evaporation process, and $R^{+}$rotons evaporate atoms with almost unit probability. $P_{+a}$ increases to 1 as the energy of the incident roton increases above the maxon, an intuitive result - above the maxon the only bulk quasiparticles are the $R^{+}$rotons. Similarly atoms with energies above the maxon (relative to bulk) condense to $R^{+}$rotons with essentially unit probability (Fig. 3).

\section{B. Oblique incidence}

\section{Fixed parallel momentum}

For a given parallel momentum, one or more quasiparticles may be excluded from the surface scattering processes at certain energies by conservation of energy and momentum parallel to the surface. In this paper we discuss parallel momenta such that phonons do not take part for some ranges of energies between the roton minimum and the maxon so as to concentrate upon roton effects.

Figure 6 shows the change in the quasiparticle dispersion, for a parallel wave vector $|\mathbf{Q}|=0.75 \AA^{-1}$, for a range of densities $\rho$ to give an indication of the change in wave vector of the quasiparticles through the surface. The important aspect is the nonmonotonic change in the energy of the phonon minimum with density. This means that effectively there is a surface barrier to penetration by atoms condensing as phonons or phonons evaporating as atoms at this parallel momentum of approximately $0.25 \mathrm{~K}$ over approximately half the surface region which, given the high effective mass for the phonon state, is significant. We believe this barrier is the origin of the structure in the probabilities (see below) in this region. The atom-phonon scattering shows a characteristic resonance maximum expected for transmission above a barrier which is coupled to enhanced transitions between various channels (Figs. 8,9,10) resulting from this surface resonance. The effective surface barrier is reflected in the 


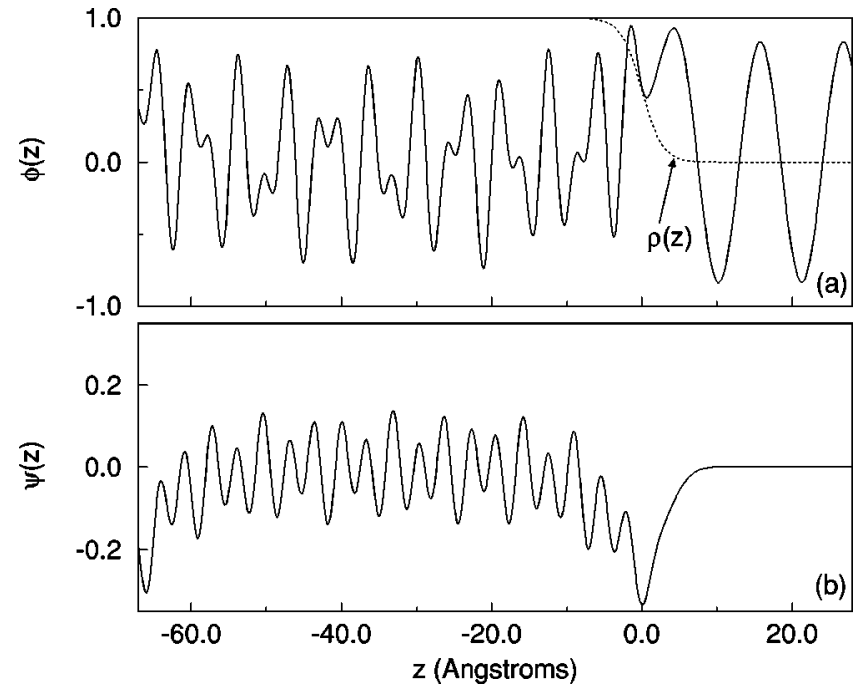

FIG. 7. The wave functions $\phi(z)$ (a) and $\psi(z)$ (b) at $\hbar \omega$ $=12.5 \mathrm{~K} .|\mathbf{Q}|=0.75 \AA^{-1}$. The short-dashed line is the surface profile.

enhanced amplitudes in the surface region of the calculated "particle-hole" wave function $\phi(z)$ and the "hole-particle", wave function $\psi(z)$ at $\hbar \omega=12.5 \mathrm{~K},|\mathbf{Q}|=0.75 \AA^{-1}$ shown, respectively, in Figs. 7(a) and 7(b) (compare Fig. 7 with Fig. 2 ). The phonon threshold at this parallel wave vector is $\Delta_{p}$ $=12.8 \mathrm{~K}$.

Figures 8, 9, 10, and 11 show the calculated probabilities $P_{i j}$ as a function of energy (relative to the zero in bulk) of the different transitions available to atoms, $R^{-}$rotons, $R^{+}$ rotons, and phonons incident on the free surface with a fixed parallel wave vector $|\mathbf{Q}|=0.75 \AA^{-1}$. As before, the probabilities satisfy both the time reversal and unitarity conditions, and conservation of energy and parallel momentum exclude phonons from the scattering processes for all energies less than the phonon threshold $\Delta_{p}=12.8 \mathrm{~K}$. Similarly there is a cutoff for propagating atom states at $\Delta_{a}=10.6 \mathrm{~K}$ (relative to bulk).

One striking feature of the results is the structure in the probabilities $P_{i j}(i, j=a,-,+)$ around the energy $\Delta_{p}$ at

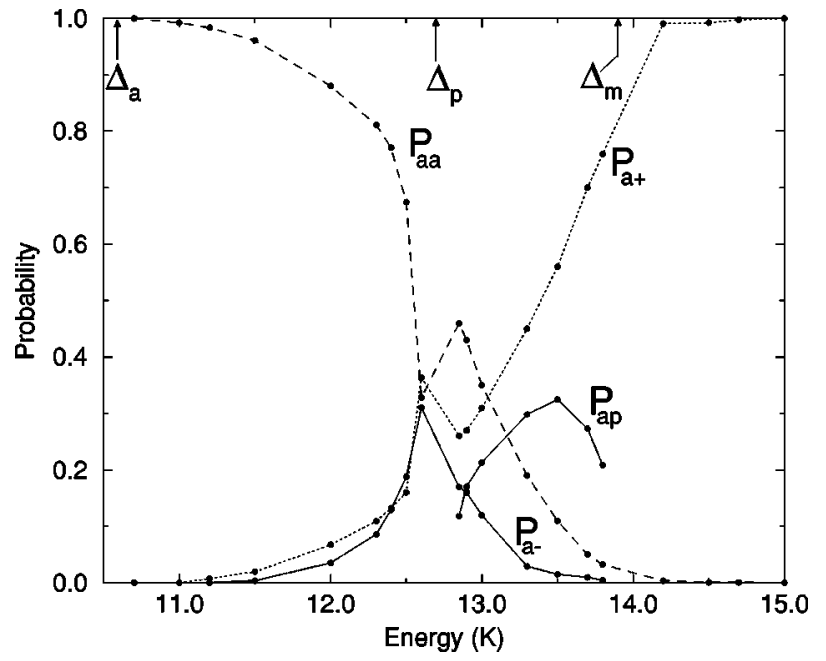

FIG. 8. The probabilities $P_{a j}$ as a function of bulk energy for an atom incident on the free surface with a parallel wave vector $|\mathbf{Q}|$ $=0.75 \AA^{-1}$.

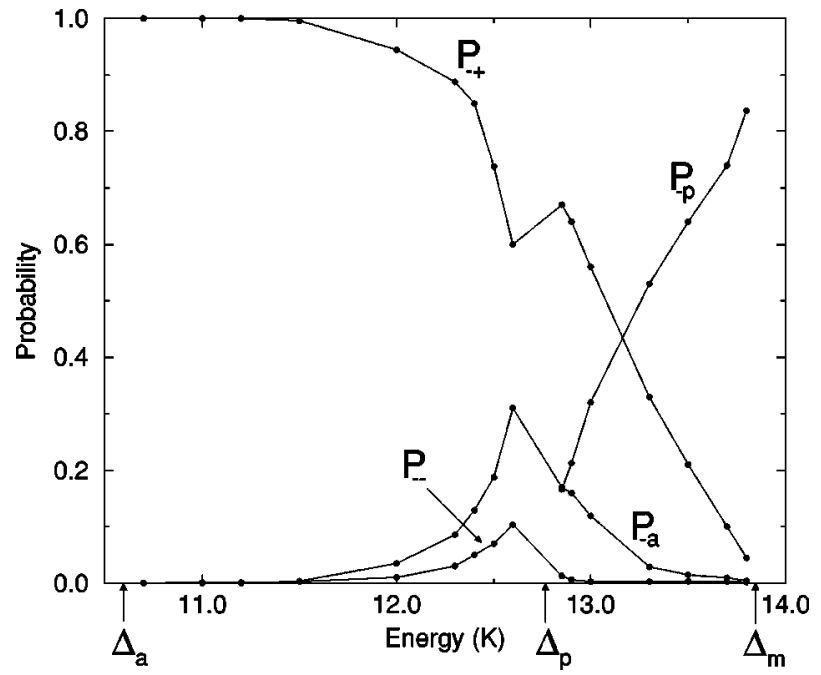

FIG. 9. The probabilities $P_{-j}$ as a function of energy for an incident $R^{-}$roton. $|\mathbf{Q}|=0.75 \AA^{-1}$.

which the phonon channel opens up on top of fairly smooth trends. This is not an artifact-calculations with other values of the parallel momenta show similar structures. Although the phonon barrier is the cause, further work on the physics associated with these features is required.

The atomic reflectivity $P_{a a}$ (Fig. 8) has the expected behavior at low energies, i.e., as the normal component $k_{a}$ of the atom wave vector tends to zero, $P_{a a}$ tends to 1 . Further as the energy of the incoming atom increases, the reflectivity decreases, but, again, we note not as fast as the measured reflectivity of Edwards et al. ${ }^{12}$ The general trend is for atoms to condense increasingly as $R^{+}$rotons though now all the three channels $R^{+}$roton, $R^{-}$roton, and phonon, play a part especially around the phonon threshold. Whereas at normal incidence atoms do not excite $R^{-}$rotons on condensing, the probability for the process is finite at oblique incidence, with $P_{a-}$ as large as $0.25-0.3$ for $|\mathbf{Q}|=0.75 \AA^{-1}$.

For incident $R^{-}$rotons (Fig. 9), the dominant transition at energies near the atom cutoff $\Delta_{a}$ is reflection as $R^{+}$rotons as expected. With increasing energy, an $R^{-}$roton tends to re-

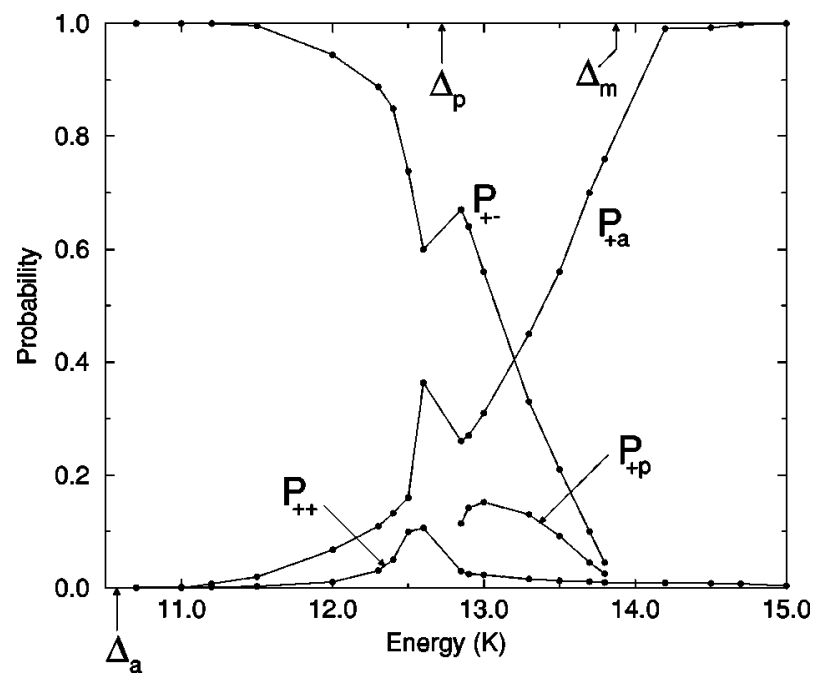

FIG. 10. The probabilities $P_{+j}$ as a function of energy for an incident $R^{+}$roton. $|\mathbf{Q}|=0.75 \AA^{-1}$. 


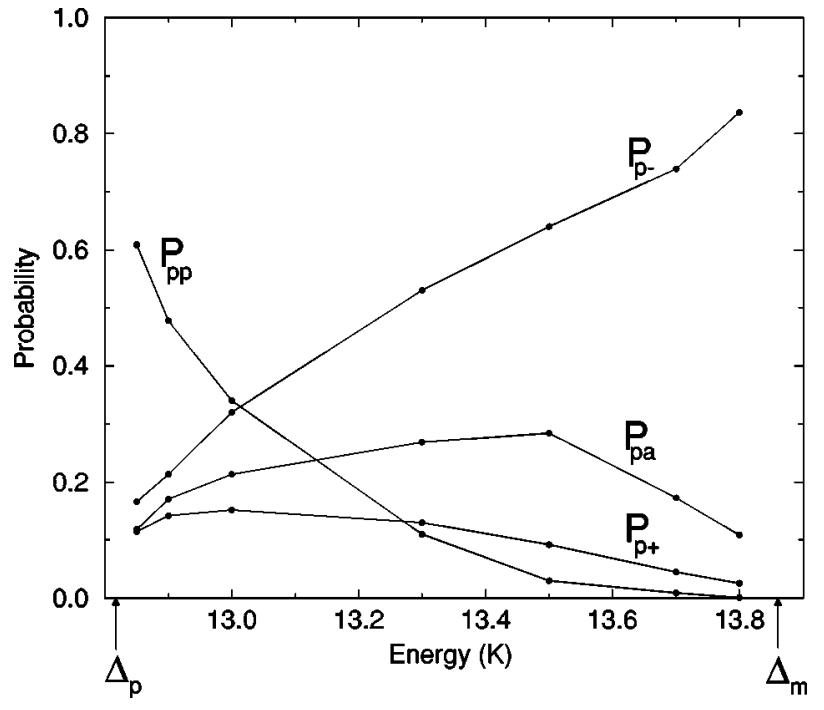

FIG. 11. The probabilities $P_{p j}$ as a function of energy for an incident phonon. $|\mathbf{Q}|=0.75 \AA^{-1}$.

flect less as an $R^{+}$roton, the dominant reflection being into a phonon at energies approaching the maxon energy. At oblique incidence, the specular reflection $R^{-}$roton to $R^{-}$roton is now possible, with $P_{--}$reaching a value just above 0.1 around $\hbar \omega=12.6 \mathrm{~K}$. The most interesting result, however, is the prediction for quantum evaporation by $R^{-}$rotons, with $P_{-a}\left(=P_{a-}\right)$ as large as $0.25-0.3$ for $|\mathbf{Q}|=0.75 \AA^{-1}$ and nonzero even above the phonon cutoff.

That $R^{-}$rotons $d o$ evaporate atoms at energies and parallel momenta at which phonons contribute in the surface scattering processes is in agreement with the recent experiments of Tucker and Wyatt ${ }^{31}$ and in contrast with the result of Dalfovo et al. ${ }^{26,27}$ who reported that $R^{-}$rotons quantum evaporate only in the phonon-forbidden regions, with the probability $P_{-a}$ dropping discontinuously to zero across the phonon cutoff. Our calculations also show that, provided the process is allowed by the conservation laws, $R^{-}$rotons evaporation is more efficient at larger parallel momenta for a given energy.

For incident $R^{+}$rotons (Fig. 10), the energy dependence of the mode-change reflection probability $P_{+-}\left(=P_{-+}\right)$and the quantum evaporation probability $P_{+a}\left(=P_{a+}\right)$ is similar to that for normal incidence. One difference, apart from the structure at $\Delta_{p}$, is that at oblique incidence the reflection $R^{+}$ roton to phonon is now possible $\left(P_{+p} \neq 0\right.$ for all energy).

For completeness, we show in Fig. 11 the probabilities for phonon scattering $P_{p i}(i=a, p,-,+)$. As mentioned earlier, for a parallel wave vector $|\mathbf{Q}|=0.75 \AA^{-1}$, phonons are only allowed in the narrow range of energy $\Delta_{p}=12.8 \mathrm{~K}<\hbar \omega$ $<\Delta_{m} \sim 13.9 \AA$. The probabilities have the same energy dependence as for normal-incidence, except for reflection into $R^{+}$rotons, which now has a finite probability.

\section{Fixed angles of incidence}

In experiments on quantum evaporation, the bolometer producing the quasiparticles is fixed at a given position in bulk helium and the beam of quasiparticles produced is collimated so that all the bulk excitations are incident to the surface at the same (fixed) angle. For a fixed angle of inci-

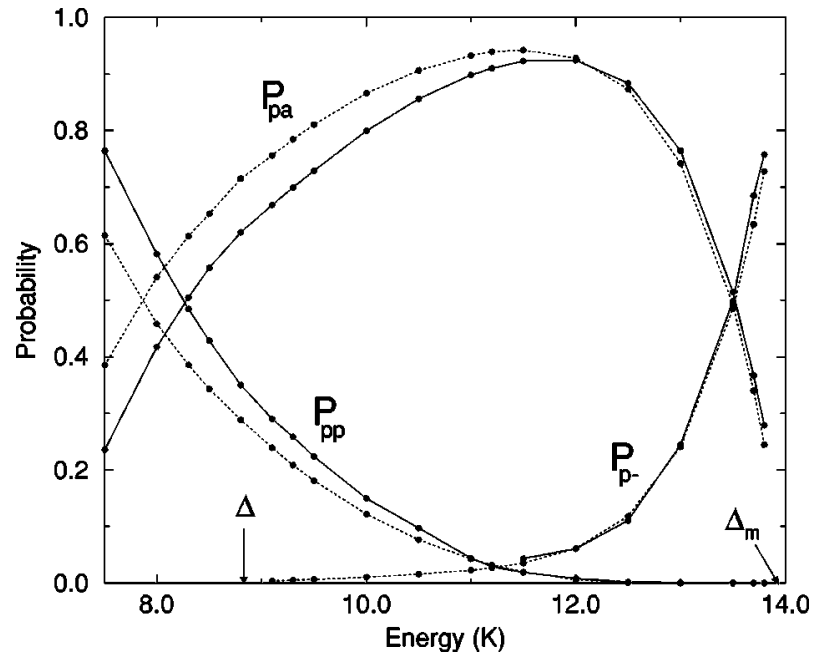

FIG. 12. The various scattering probabilities $P_{p j}$ as a function of bulk energy for a phonon incident on the surface at $\theta=14^{\circ}$ (dotted lines) and $\theta=25^{\circ}$ (solid lines). $\Delta$ and $\Delta_{m}$ are the roton minimum energy and the maxon energy, respectively.

dence $\theta_{i}$, different excitations incident on the surface have different parallel momenta $\hbar \mathbf{Q}\left(\theta_{i}, \hbar \omega\right)$. We have calculated the scattering rates for fixed angles of incidence, $\theta_{i}=14^{\circ}$ and $\theta_{i}=25^{\circ}$ to enable comparison of our results with experiments.

We plot our calculated probabilities for phonons, $R^{-}$rotons and $R^{+}$rotons incident at $\theta_{i}=14^{\circ}$ (dotted line) and $\theta_{i}$ $=25^{\circ}$ (solid line) in Figs. 12, 13, and 14, respectively. From consideration of the conservation laws, we know that for energies $\hbar \omega$ less than about $11.5 \mathrm{~K}$ there are no phonons with parallel momentum large enough to come in at $25^{\circ}$. Similarly $R^{-}$rotons incident at $25^{\circ}$ cannot evaporate atoms at energies less than the atom cutoff $\Delta_{a}\left(25^{\circ}\right)$ and cannot reflect as phonons at energies less than the phonon cutoff $\Delta_{p}\left(25^{\circ}\right)$, and $R^{+}$rotons coming in with energy $\hbar \omega$ cannot

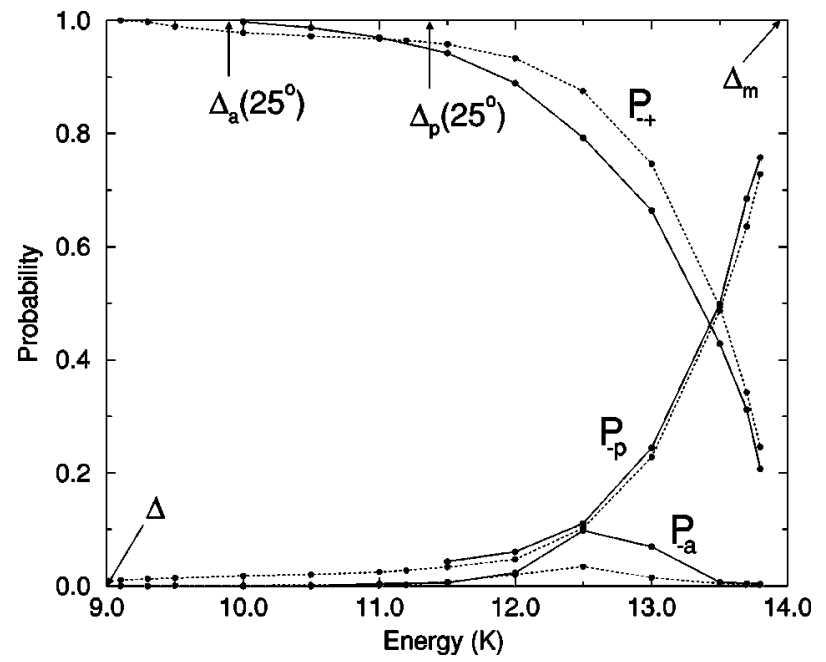

FIG. 13. The various scattering probabilities $P_{-j}$ as a function of bulk energy for an $R^{-}$roton incident on the surface at $\theta=14^{\circ}$ (dotted lines) and $\theta=25^{\circ}$ (solid lines). $\Delta_{a}\left(25^{\circ}\right)$ and $\Delta_{p}\left(25^{\circ}\right)$ are, respectively, the atom and phonon threshold when $R^{-}$rotons are incident at $25^{\circ} . \Delta$ and $\Delta_{m}$ are the roton minimum energy and the maxon energy, respectively. 


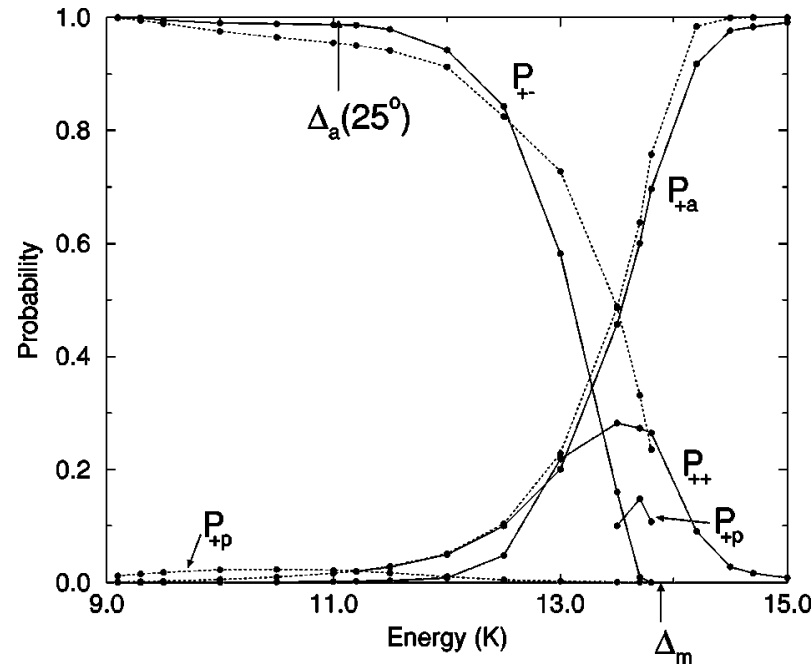

FIG. 14. The various scattering probabilities $P_{+j}$ as a function of bulk energy for a phonon incident on the surface at $\theta=14^{\circ}$ (dotted lines) and $\theta=25^{\circ}$ (solid lines). $\Delta_{m}$ is the maxon energy.

evaporate atoms for $\hbar \omega$ less than about $11.1 \mathrm{~K}$ and cannot excite phonons for $\hbar \omega$ less than about $13.6 \mathrm{~K}$.

The results show that the calculated probabilities have a definite angular dependence. In particular, the probability $P_{p p}$ of a phonon reflecting as a phonon or the probability $P_{p a}$ of evaporating an atom depends quite strongly on the angle of incidence for energies up to about $12 \mathrm{~K}$ (Fig. 12). The mode change reflection phonon to $R^{-}$roton, however, is independent of the angle of incidence. One possible explanation is that the change in (normal) momentum involved in this reflection does not depend very strongly on the angle of incidence (for angles and energies such that the process is allowed).

Again, for an incoming $R^{-}$roton (Fig. 13), the probability $P_{-p}$ of the reverse reflection is independent of the angle of incidence, whereas the probability of the incident quasiparticle reflecting as an $R^{-}$roton or evaporating an atom is not. For instance, $R^{-}$rotons incident at $25^{\circ}$ are at least twice as likely to quantum evaporate than those incident at $14^{\circ}$. This enormous variation in the $R^{-}$roton evaporation probability comes at the expense of only a $10 \%$ decrease in the $R^{-}$to $R^{+}$scattering channel. We again note that $P_{-a}$ is nonzero for energies $\hbar \omega>\Delta_{p}\left(25^{\circ}\right)$ showing that the presence of phonons does not inhibit $R^{-}$rotons from evaporating atoms in contrast with the result of Dalfovo et al..$^{26,27}$

The probability $P_{+a}$ of quantum evaporation by $R^{+}$rotons is independent of the angle of incidence (Fig. 14), but the reflections $R^{+}$roton to phonons and to $R^{-}$rotons change markedly with the angle of incidence.

All the above results differ significantly from those of Dalfovo and co-workers, ${ }^{30}$ who reported that their calculated probabilities do not depend significantly on the angle of incidence, apart from threshold effects.

There are no direct measurements of $P_{i j}$ with which to compare our results. However, our calculated evaporation probabilities have been used ${ }^{33,40}$ together with experimental evaporated atom signal data from time of flight experiments in numerical simulations.

The published experiments inject a distribution $n_{i}(k)$ of ballistic excitations $i$ in the liquid by pulsing a thin-film

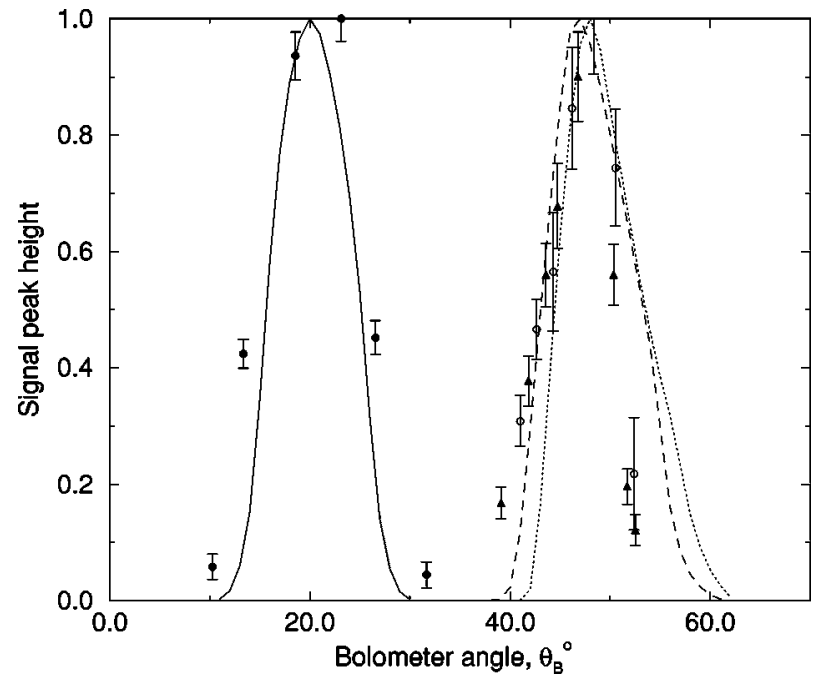

FIG. 15. The angular dependence of the peak height of the measured (points) and simulated (curves) phonon $\rightarrow$ atom signals for angles of incidence of $\theta=25^{\circ}$ (full circles, solid curve), $75^{\circ}$ (full triangles, long dashes), and $83^{\circ}$ (open circles, short dashes). Each distribution is normalized to the maximum data point [after Williams (Ref. 40)]. See also Fig. 6 of Brown and Wyatt (Ref. 8).

heater and, by detecting evaporated atoms, measure the relative size of the product $P_{i a}(k) n_{i}(k) .{ }^{8}$ Recently Tucker and Wyatt ${ }^{41}$ have independently measured the distribution $n_{p}(k)$ of high-energy phonons $(\hbar \omega \sim 10 \mathrm{~K})$ and this has prompted Williams ${ }^{40}$ to reanalyze the experiments and to eliminate some sources of systematic error. For high-energy phonons, the corrected model, which assumes that $P_{p a}(k)$ is constant $(\sim 0.3)$, agrees with the mechanical accuracy of the experiments. Use of our calculated phonon evaporation probabilities $P_{p a}$ for phonons incident at $\theta_{i}=25^{\circ}$ (solid line in Fig. 12) does not change the solid curve in Fig. 15 by a significant amount. ${ }^{40}$ Finer details, ${ }^{40}$ however, show that our calculations probably overestimate the phonon-atom probability. A proper test of our calculated phonon evaporation probabilities will require a direct measurement of the evaporation probability, or a source that generates a much wider phonon spectrum.

There have been no successful attempts to measure the distribution $n_{+}(k)$ of injected $R^{+}$rotons. Brown and $\mathrm{Wyatt}^{8}$ modelled their experiments assuming some thermal distribution for $n_{+}(k)$,

$$
n_{+}(k) d k \propto \frac{k^{\lambda} d k}{\exp \left(\hbar \omega / T_{\mathrm{eff}}-1\right)} \text { with } \lambda=2 .
$$

This distribution is dominated by the value of the parameter $T_{\text {eff }}{ }^{40}$ Figure 16 shows the results of the numerical simulations of Williams ${ }^{40}$ using the evaporated atom signals of Brown and Wyatt: ${ }^{8}$ the thin lines assume that $P_{+a}=1$ and the thick lines uses our calculated $R^{+}$roton evaporation probabilities (shown in Fig. 14). The simulations show that, while our calculated evaporation probability $P_{+a}$ has the correct energy dependence (i.e., it tends to 1 as the energy of the incident roton approaches the maxon energy), it rises too sharply with energy - it is too small at lower energies. There are, at present, insufficient data for atom signals due to evaporation by $R^{-}$rotons and, furthermore, the distribution 


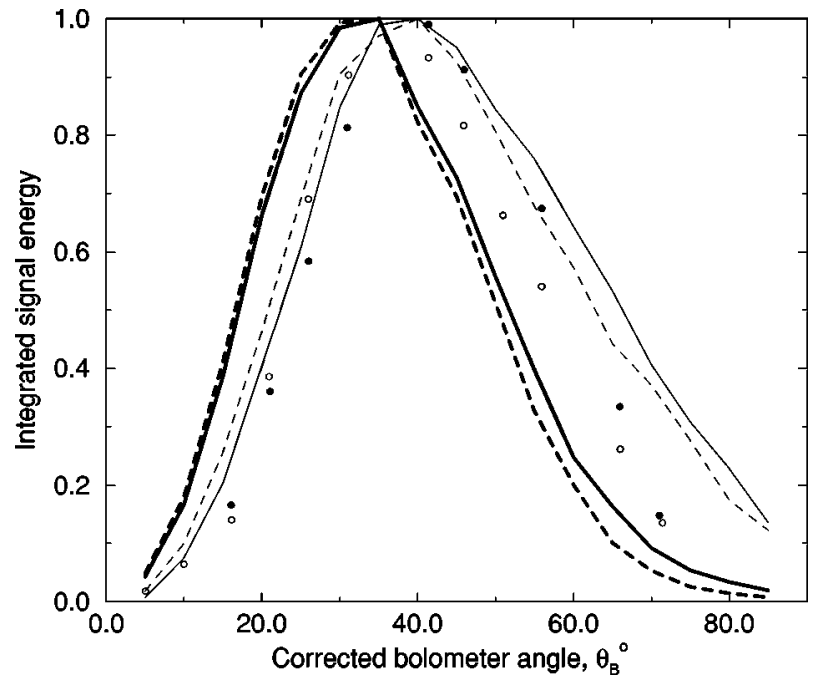

FIG. 16. The angular dependence of the integrated $R^{+} \rightarrow$ atom signal energy. The angle of incidence is $\theta=14^{\circ}$. The signal are integrated up to $160 \mu$ s after the start of the heater input pulse. The points are experiments using two different heater powers, $-27 \mathrm{~dB}$ (full circles) and $-24 \mathrm{~dB}$ (open circles). The curves are simulations using injected-roton spectra at two characteristic temperatures, $T_{\text {eff }}$ $=1.0 \mathrm{~K}$ (solid lines) and $T_{\text {eff }}=1.5 \mathrm{~K}$ (dashed lines). The thin lines assume $P_{+a}=$ const and the thick lines use the values reported in this work [after Williams (Ref. 40)]. See also Fig. 8 of Brown and Wyatt (Ref. 8).

of $R^{-}$rotons produced by the heater is still unknown to enable one to do similar simulations.

\section{SUMMARY AND CONCLUDING REMARKS}

We have presented a method for studying the scattering of quasiparticles at the free surface of superfluid ${ }^{4} \mathrm{He}$. The numerical solutions to the equations of motion for the quasiparticle wave functions are stable and accurate. The probabilities $P_{i j}$ for the one-to-one scattering processes calculated from the current associated with the wave functions satisfy both the time-reversal symmetry and the unitarity conditions, and can be understood in terms of the change in the nature of the states through the surface region.

In particular, our results show that atoms impinging on the surface have a finite probability of reflecting in the limit of small atom normal wave vector both for normal incidence and oblique incidence, in agreement with both experiments ${ }^{12}$ and basic scattering theory. The probability, however, does not fall as rapidly with energy as the experiments suggest and it is reasonable that atom condensation through processes such as two-phonon production would reduce the probability at high energies.

The results also show that at oblique incidence all transitions allowed by energy and parallel momentum conservation requirements take place whereas at normal incidence certain channels are not excited (within our calculation accuracy). For instance, while $R^{-}$rotons do not quantum evaporate and atoms do not condense as $R^{-}$rotons at normal incidence, these channels open up as the parallel momentum increases. Calculations at energies and parallel momenta which do not exclude phonons from the scattering processes show that $R^{-}$rotons do evaporate atoms in the presence of phonons, in agreement with the recent experiments of Wyatt and Tucker. ${ }^{31}$ However $R^{-}$rotons are always less efficient at quantum evaporating that $R^{+}$rotons.

Experimental simulations using our calculated evaporation probabilities show very good agreement with experiments for phonons, but show that our model underestimates the efficiency of evaporation by $R^{+}$rotons at low energies. This suggests possibly the need for a better description of the roton-one, for example, that includes backflow. Inclusion of backflow effects and inelastic processes into the present formalism is difficult, but important, and work along these lines are in progress.

\section{ACKNOWLEDGMENTS}

M.B.S. would like to acknowledge financial support from the EPSRC, and J.C.H.F. and M.B.S. financial support from the HKUST (Grant No. DAG95/96.SC17). M.B.S. is grateful to M. A. H. Tucker, C. A. Jones, and J. R. Matthias for many invaluable discussions and to C. D. H. Williams for permission to use his analysis ${ }^{40}$ of our results.

${ }^{1}$ W. D. Johnston, Jr. and J. C. King, Phys. Rev. Lett. 16, 1191 (1966).

${ }^{2}$ P. W. Anderson, Phys. Lett. 29A, 563 (1969).

${ }^{3}$ D. S. Hyman, M. O. Scully, and A. Widom, Phys. Rev. 186, 231 (1969).

${ }^{4}$ S. Balibar, J. Buechner, B. Castaing, C. Laroche, and A. Libchaber, Phys. Rev. B 18, 3096 (1977).

${ }^{5}$ M. J. Baird, F. R. Hope, and A. F. G. Wyatt, Nature (London) 304, 325 (1983).

${ }^{6}$ A. F. G. Wyatt, Physica B 126, 392 (1984).

${ }^{7}$ G. M. Wyborn and A. F. G. Wyatt, Jpn. J. Appl. Phys., Suppl. 26-3, 2095 (1987).

${ }^{8}$ M. Brown and A. F. G. Wyatt, J. Phys.: Condens. Matter 2, 5025 (1990).

${ }^{9}$ A. F. G. Wyatt, in Phonons 89 , edited by S. Hunklinger, W. Ludwig, and G. Weiss (World Scientific, Singapore, 1990), p. 1019.

${ }^{10}$ M. A. H. Tucker and A. F. G. Wyatt, J. Low Temp. Phys. 110, 455 (1998)

${ }^{11}$ G. M. Wyborn and A. F. G. Wyatt, Phys. Rev. Lett. 65, 345 (1990).

${ }^{12}$ D. O. Edwards, P. P. Fatouros, G. G. Ihas, P. M. Rozinsky, S. Y. Shen, F. M. Gasparini, and C. P. Tam, Phys. Rev. Lett. 34, 1153 (1975).

${ }^{13}$ P. M. Echenique and J. B. Pendry, Phys. Rev. Lett. 37, 561 (1976).

${ }^{14}$ P. M. Echenique and J. B. Pendry, J. Phys. C 9, 3183 (1976).

${ }^{15}$ D. O. Edwards, G. G. Ihas, and C. P. Tam, Phys. Rev. B 16, 3122 (1977).

${ }^{16}$ D. O. Edwards and P. P. Fatouros, Phys. Rev. B 17, 2147 (1977).

${ }^{17}$ D. R. Swanson and D. O. Edwards, Phys. Rev. B 37, 1539 (1988).

${ }^{18}$ C. Caroli, B. Roulet, and D. Saint-James, Phys. Rev. B 13, 3875 (1976). 
${ }^{19}$ C. Caroli, B. Roulet, and D. Saint-James, Phys. Rev. B 13, 3884 (1976).

${ }^{20}$ S. Sasaki and T. Kunimasa, J. Phys. Soc. Jpn. 58, 3651 (1989).

${ }^{21}$ A. Griffin, Phys. Lett. 31A, 222 (1970).

${ }^{22}$ M. W. Cole, Phys. Rev. Lett. 28, 1622 (1972).

${ }^{23}$ H. J. Maris, J. Low Temp. Phys. 87, 773 (1992).

${ }^{24}$ F. R. Hope, M. J. Baird, and A. F. G. Wyatt, Phys. Rev. Lett. 52, 1528 (1984).

${ }^{25}$ P. A. Mulheran and J. C. Inkson, Phys. Rev. B 46, 5454 (1992).

${ }^{26}$ F. Dalfovo, A. Fracchetti, A. Lastri, L. Pitaevskii, and S. Stringari, J. Low Temp. Phys. 104, 367 (1996).

${ }^{27}$ F. Dalfovo, A. Fracchetti, A. Lastri, L. Pitaevskii, and S. Stringari, Phys. Rev. Lett. 75, 2510 (1995).

${ }^{28}$ S. Stringari, F. Dalfovo, M. Guilleumas, A. Lastri, and L. Pitaevskii, Czech. J. Phys. 46-S6, 2973 (1996).

${ }^{29}$ F. Dalfovo, M. Guilleumas, A. Lastri, L. Pitaevskii, and S. Stringari, J. Phys.: Condens. Matter 9, L369 (1997).

${ }^{30}$ M. Guilleumas, F. Dalfovo, I. Oberosler, L. Pitaevskii, and S.
Stringari, J. Low Temp. Phys. 110, 449 (1998).

${ }^{31}$ M. A. H. Tucker and A. F. G. Wyatt (unpublished).

${ }^{32}$ M. B. Sobnack and J. C. Inkson, Phys. Rev. B 56, R14 271 (1997).

${ }^{33}$ M. B. Sobnack and J. C. Inkson, Phys. Rev. Lett. 82, 3657 (1999).

${ }^{34}$ N. N. Bogoliubov, J. Phys. (Moscow) 11, 23 (1947).

${ }^{35}$ S. T. Beliaev, Sov. Phys. JETP 7, 289 (1958).

${ }^{36}$ N. Hugenholtz and D. Pines, Phys. Rev. 116, 489 (1959).

${ }^{37}$ D. Pines, The Many-Body Problem (Benjamin, New York, 1962), p. 80.

${ }^{38}$ K. A. Brueckner and K. Sawada, Phys. Rev. 106, 1117 (1957); 106, 1128 (1957).

${ }^{39}$ D. V. Osborne, J. Phys.: Condens. Matter 1, 289 (1989).

${ }^{40}$ C. D. H. Williams, J. Low Temp. Phys. 113, 627 (1998).

${ }^{41}$ M. A. H. Tucker and A. F. G. Wyatt, J. Phys.: Condens. Matter 6, 2813 (1994); 6, 2825 (1994). 